\title{
Identifying equatorial ionospheric irregularities using in situ ion drifts
}

\author{
R. A. Stoneback and R. A. Heelis \\ W. B. Hanson Center for Space Sciences, Physics Department, University of Texas at Dallas, Richardson, Texas, USA
}

Correspondence to: R. A. Stoneback (rstoneba@utdallas.edu)

Received: 16 November 2013 - Revised: 4 February 2014 - Accepted: 15 February 2014 - Published: 15 April 2014

\begin{abstract}
Previous climatological investigations of ionospheric irregularity occurrence in the equatorial ionosphere have utilized in situ measurements of plasma density to identify the presence of an irregularity. Here we use the Morlet wavelet and C/NOFS to isolate perturbations in meridional ion drifts and generate irregularity occurrence maps as a function of local time, longitude, season, and solar activity. For the low solar activity levels in 2008 , the distributions identified by velocity perturbations follow normalized density perturbation $(\Delta N / N)$ maps with large occurrences after midnight into dawn over all longitudes. The velocity and normalized density occurrence maps contract in both local time and longitude with increasing solar activity. By 2011 irregularities are confined to particular longitudes expected by alignment and a few hours of local time after sunset. The variation in the occurrence of the late night irregularities with solar activity is consistent with the presence of gravity wave seeding.
\end{abstract}

Keywords. Ionosphere (equatorial ionosphere; ionosphereatmosphere interactions; ionospheric irregularities)

\section{Introduction}

Irregularities in the plasma number density are a common feature of the equatorial ionosphere that can be responsible for significant disruption to radio communication and navigation systems. The irregularities are born in the bottom side of the $\mathrm{F}$ region and grow non-linearly during the night through the generation of polarization fields that cannot be shorted through the underlying E region.

The exact mechanisms that underlie irregularity formation are still a topic of study (review by Abdu, 2012), though the generalized Rayleigh-Taylor instability is generally agreed to play a role in irregularity formation. This instability is dependent upon the ion-neutral collision frequency; thus, an upward vertical $\boldsymbol{E} \times \boldsymbol{B}$ ion drift, which raises the height of the ionosphere into lower neutral densities, is thought to play an important role in irregularity formation (Sultan, 1996). The strength and duration of the pre-reversal enhancement (PRE), which usually occurs near sunset, is influential in determining the magnitude and duration of the irregularities that develop (Abdu et al., 1981; Stolle et al., 2008).

Other instability processes may also be a factor; Aveiro et al. (2012) combined the collisional shear instability and the generalized Rayleigh-Taylor instability numerically and found that irregularities were produced that displayed underlying characteristics of 30 and $100 \mathrm{~km}$ waves. The collisional shear instability is driven by a shear in the relative zonal ion neutral drift with altitude near sunset. In the topside $F$ region plasma will drift westward with the neutral atmosphere while at lower altitudes plasma will drift eastward against the neutral flow. The numerical model was seeded with white noise; thus the 30 and $100 \mathrm{~km}$-scale size perturbations that develop are initially supplied to the model, among many others. Significantly, however, the scale sizes that dominate are not preferentially supplied to the numerical model.

Seasonal and longitudinal variations in the occurrence of irregularities are expected to follow variations in the main drivers of irregularities. The alignment of the magnetic meridian with the sunset terminator has a large influence on the integrated conductance of a field line. The lowaltitude portions of field lines aligned with the terminator cross into darkness simultaneously increasing conductivity gradients with local time. The gradient in conductivity across the terminator has an effect on the magnitude of the fringing field from the $\mathrm{F}$ region dynamo that leads to the PRE (Abdu et al., 1981; Eccles, 1998). Numerous satellite investigations 
(Kil and Heelis, 1998a; Hei et al., 2005; Gentile et al., 2006; Su et al., 2006; Stolle et al., 2008; Kil et al., 2009; Heelis et al., 2010; Dymond, 2012) have shown that a component of the seasonal and longitudinal variations in irregularity occurrence may be explained by the alignment of the terminator and magnetic field (Tsunoda, 1985).

Seasonal distributions of irregularities have been formed using in situ satellite measurements of density that identify irregularities using sharp changes or a large variance in density. Here we use the Morlet wavelet and the continuous wavelet transform to isolate perturbations in meridional ion drift as a function of scale size to identify irregularities measured by the Coupled Ion Neutral Dynamics Investigation (CINDI) on board the Communication and Navigation Outage Forecasting System (C/NOFS). For comparison, similar distributions are computed using perturbations in density $(\Delta N)$ and normalized density $(\Delta N / N)$. The density perturbation calculation follows the process detailed by Stoneback et al. (2013), which is extended to velocity measurements. The process for constructing $\Delta N / N$ is presented here. The occurrence of irregularities using each of these indices is presented as a function of local time, longitude, solar activity, and season. The in situ results are validated against seasonal climatologies of COSMIC estimates of scintillation.

$\mathrm{Su}$ et al. (2008) concluded that the vertical ion drift velocity was the best indicator for the formation of irregularities using ROCSAT-1. Stolle et al. (2008) found that integrating the vertical ion drift measured by ROCSAT-1 during the PRE was a better indicator of irregularity formation as measured by CHAMP but did not rule out the peak vertical drift as a leading indicator as concluded by Su et al. (2008). We find at moderate levels of solar activity that irregularity formation after sunset is consistent with the upward drift at sunset. However, at solar minimum irregularities are found over all longitudes with little relationship to the alignment of the solar terminator with the magnetic field. The occurrence of irregularities decreases smoothly with increasing solar flux absent changes in the ion drift until moderate activity levels are reached. The observed dependence in longitude and solar activity is consistent with seeding from gravity waves and the reduction in force applied by a gravity wave with increasing solar activity (Vadas and Fritts, 2006).

\section{Data}

The C/NOFS satellite was launched in April, 2008 into an equatorial orbit with $13^{\circ}$ inclination. The orbit is elliptical with perigee originally at $400 \mathrm{~km}$ and apogee at $850 \mathrm{~km}$. Ion measurements by CINDI are performed by two instruments: a retarding potential analyzer (RPA) and an ion drift meter (IDM). The RPA measures plasma temperature, density, composition, and ion drift velocity along the satellite track while the IDM measures ion drift velocities in two orthogonal directions perpendicular to the satellite track. The full three-dimensional velocity vector is transformed into a magnetic coordinate system using GEOPACK-2008 (Tsyganenko, 2008) and the attitude and position of C/NOFS. The meridional $\boldsymbol{E} \times \boldsymbol{B}$ ion drift utilized here is perpendicular to the magnetic field and in the plane of the magnetic meridian. Positive drifts are vertically up at the geomagnetic equator.

The FormoSat-3 constellation COSMIC (Anthes et al., 2008) is a constellation of six micro-satellites launched in April, 2006, each carrying a GPS receiver (Schreiner et al., 2012). Measurements of GPS signals as they occult the ionosphere may be converted to electron density profiles using the Abel inversion (Lei et al., 2007). Assumptions with this inversion break down when there are large horizontal gradients in the ionosphere. Errors may be found within the $\mathrm{E}$ region as well as at low latitudes. The derived parameters $N m \mathrm{~F} 2$ and $h m \mathrm{~F} 2$ are less affected by these errors, and are generally reliable (Yue et al., 2010). Profiles are restricted to derived $h m F 2$ altitudes between 175 and $475 \mathrm{~km}$. The profiles provided by the COSMIC Data Analysis and Archival Center (CDAAC) may also contain errors due to cycle slips or multi-path signals (Hwang et al., 2010). These distorted profiles are excluded by filtering profiles with sharp density gradients.

Variances in the signal during the RO may also be used to estimate the scintillation index (Ko, 2010; Dymond, 2012). Seasonal occurrence frequencies for scintillation are computed using COSMIC data for comparison to the CINDI in situ irregularity estimate. We use the averaged $S_{4}$ index over $9 \mathrm{~s}$ around the maximum $S_{4}$ for a given RO, publicly available at CDAAC. Though scintillation effects may occur anywhere along the GPS signal path, the location of the scintillation is taken to be the tangent ray height following Straus et al. (2003) and Dymond (2012).

In situ measurements of plasma density and meridional ion drift by CINDI are analyzed and processed using the Morlet wavelet (Torrence and Compo, 1998). The Morlet wavelet is constructed using a combination of sine and cosine functions, windowed in time by a Gaussian function,

$\Psi_{\circ}(\eta)=\pi^{-1 / 4} e^{-i \omega_{\circ} \eta} e^{-\eta^{2} / 2}$,

where $\eta$ is a non-dimensional "time" parameter. The nondimensional frequency $\omega_{\circ}$ is taken to be six to satisfy the admissibility condition for wavelets. The wavelet transform is determined by the convolution of the wavelet with the desired signal.

The use of sines and cosines gives the Morlet wavelet decomposition an interpretation similar to the Fourier transform; however, the use of a Gaussian time window provides for greater specificity in time. This wavelet has been used by Stoneback et al. (2013) to determine a scale-limited variance in ion density. Changes in this wavelet-determined variance are consistent with ground-based measurements of scintillation by SCINDA (Stoneback et al., 2013) and are shown here to be consistent with climatological measurements by COSMIC (FormoSat-3). 
The variation in altitude for C/NOFS leads to measured variations in ion density that should not be confused with irregularity formation; thus, the wavelet variance is limited here to a maximum scale size of $100 \mathrm{~km}$. The relationship between the wavelet scale size $(s)$ and Fourier wavelength $(\lambda)$ is wavelet dependent. For the Morlet configuration used here, the two are nearly equal (Torrence and Compo, 1998),

$s=1.03 \lambda$.

The scale-limited variance is calculated following (Torrence and Compo, 1998)

$|\overline{\Delta A}|^{2}=\frac{\delta j \delta t}{C} \sum_{j=0}^{J} \frac{P_{j}}{s_{j}}$,

where $\overline{\Delta A}$ is the variance at a given time, $P_{j}$ is the wavelet power at size $s_{j}, \delta t$ is the time between samples, $\delta j$ is the dyadic spacing between scale sizes $(0.25)$, and $C$ is a constant dependent upon the wavelet choice. This equation is used to obtain the variance in plasma density $(\overline{\Delta N})$ and the meridional ion $\operatorname{drift}(\overline{\Delta V})$.

The normalized density variation $\Delta N / N$ is the change in ion density normalized by the background plasma density. The change in density is obtained by calculating the waveform associated with a limited scale range from the wavelet transform

$\Delta N=\frac{\delta j \delta t^{1 / 2}}{C \psi_{\circ}(0)} \sum_{j=0}^{J} \frac{\operatorname{Re}\left(W_{n}\left(s_{j}\right)\right)}{s_{j}^{1 / 2}}$,

where $\operatorname{Re}\left(W_{n}(s)\right)$ is the real part of the wavelet transform and $\psi_{\circ}(0)$ is a wavelet-dependent scalar. The background ionosphere from which irregularities deviate is estimated by subtracting the perturbation waveform from the raw measurements,

$N_{\text {bkgd }}=N_{\text {raw }}-\Delta N$.

Scale sizes up to $850 \mathrm{~km}$ are used to calculate $\Delta N$ to provide a more accurate estimate of the background ionosphere. The absolute normalized density change is thus

$\frac{|\Delta N|}{N}=\frac{|\Delta N|}{N_{\text {raw }}-\Delta N}$,

where $\Delta N$ in the numerator and denominator are both given by Eq. (4). Alternately, the variance in density $(\overline{\Delta N})$ could be used in the numerator. A larger scale range may be tolerated when only utilizing the real part of the wavelet transform as it couples most strongly with perturbations centered on the time investigated. The wavelet power used in the variance also includes a contribution from the imaginary component, or sinusoidal term, which maximizes with an oscillation out of phase with the current time. This increases the temporal range over which a perturbation at a given scale size and time will produce an elevated response.
To limit the impact of altitude variations in the C/NOFS orbit upon the sampling of irregularities, measurements are restricted to altitudes less than $550 \mathrm{~km}$ for all seasons. Measurements are also restricted to satellite locations within $12^{\circ}$ of the magnetic equator and to periods of low magnetospheric activity such that the Kp index must be less than $3+$ for at least $24 \mathrm{~h}$. Results do not change significantly if the maximum allowed $\mathrm{Kp}$ is altered or even removed.

The $\overline{\Delta N}, \overline{\Delta V}$, and $\Delta N / N$ are used to generate occurrence probability distributions as a function of local time, apex longitude, season, and solar activity. An irregularity is identified when the relevant wavelet-derived index passes a threshold, $\overline{\Delta N}>5 E 3 N / \mathrm{cc}, \overline{\Delta V}>5 \mathrm{~m} \mathrm{~s}^{-1}$, or $\Delta N / N>0.15$. The occurrence of irregularities is defined as the number of C/NOFS orbits that encounter an irregularity divided by the number of orbits that pass through that bin (Kil et al., 2009). We will show these thresholds produce irregularity maps consistent with COSMIC estimates of scintillation.

Apex longitude is used to organize the data according to the dominant dipole structure of the magnetic field, shown in Fig. 1. Gravity waves are driven by processes in the neutral atmosphere, such as weather patterns, that are organized geographically. However, the effects of gravity waves upon irregularity formation will map along field lines and are thus influenced by the magnetic field. Irregularity formation is also expected to be influenced by the alignment of the sunset terminator and the magnetic field. This situation results in some tension between the most appropriate coordinate system for analysis. Apex coordinates are used here so that declination effects are most clearly identified, allowing for simple identification of irregularities that deviate from this pattern. To ensure the choice of coordinates does not unduly impact any of the conclusions presented, measurements were analyzed in both systems and found to be consistent. The presented seasonal averages may be approximately translated into geographic longitudes by adding $-72^{\circ}$ to the listed apex longitudes.

\section{Results}

The wavelet-derived indices for identifying the presence of an irregularity are shown in Fig. 2 for a single pass. The threshold value for each index is shown with a dashed line. In the top panel the raw density measurements are shown along with the wavelet-derived variance in density, $\overline{\Delta N}$. Note how the density perturbations are low until the appearance of irregularities. The middle panel shows both the average density $(N-\Delta N)$ and the resulting normalized density change, $\Delta N /(N-\Delta N)$. Since the Morlet wavelet is oscillatory, the average density obtained tends to travel through the middle of the wider density perturbations. Both the absolute and normalized density indices indicate irregularities at the same locations. The bottom panel has measurements of the meridional ion drift along with the wavelet-determined variance 


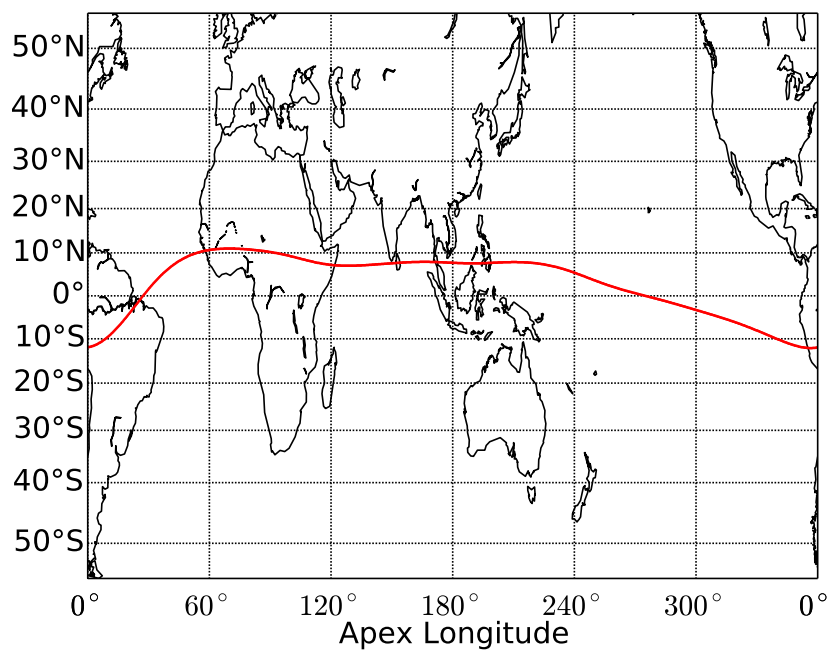

Fig. 1. Map of the Earth as a function of apex longitude and geographic latitude overlaid with the geomagnetic equator in red as determined by the International Geomagnetic Reference Field . $0^{\circ}$ apex longitude is approximately $-72^{\circ}$ geographic longitude.

$(\overline{\Delta V})$. Like the other indices, the magnitude is low before the onset of irregularities and increases with the presence of an irregularity. However, one irregularity does not have a velocity variance above the $5 \mathrm{~m} \mathrm{~s}^{-1}$ threshold.

Figure 3 shows the irregularity occurrence distribution as a function of apex longitude and magnetic local time (MLT) for the December solstice seasons, defined as 134 days centered upon the solstice. The mean solar activity level for each season is listed in Table 1. Each row in the figure is a separate year, and the columns vary the wavelet product used to construct the distribution. The first column is the $\overline{\Delta N}$ occurrence distribution and tends to exhibit the narrowest range of longitudes and local times. The large reduction in the background density for the nighttime ionosphere as a function of local time tends to inhibit a $\overline{\Delta N}$ optimized for sunset from identifying irregularities late at night. The same longitudinal distribution is seen for all seasons with a maximum occurrence east of $0^{\circ}$ apex longitude, or $-72^{\circ}$ geographic longitude. The maximum occurrence in longitude is coincident with areas of negative magnetic declination, consistent with the alignment hypothesis (Abdu et al., 1981; Tsunoda, 1985). The largest occurrences are seen in the December solstice of 2011, coincident with the largest solar activity levels and the highest ambient density levels.

The $\Delta N / N$ distributions in the second column show irregularities over a significantly larger local time and longitudinal range than for $\Delta N$. The distribution for December 2008 is consistent with a similar plot by Heelis et al. (2010) that used a running mean over $400 \mathrm{~km}$ to determine $\Delta N / N$. Immediately after sunset, the distribution follows $\overline{\Delta N}$; however, it quickly expands westward into an area with positive magnetic declination. As solar activity increases, the late night irregularities decrease smoothly in occurrence, and by 2011

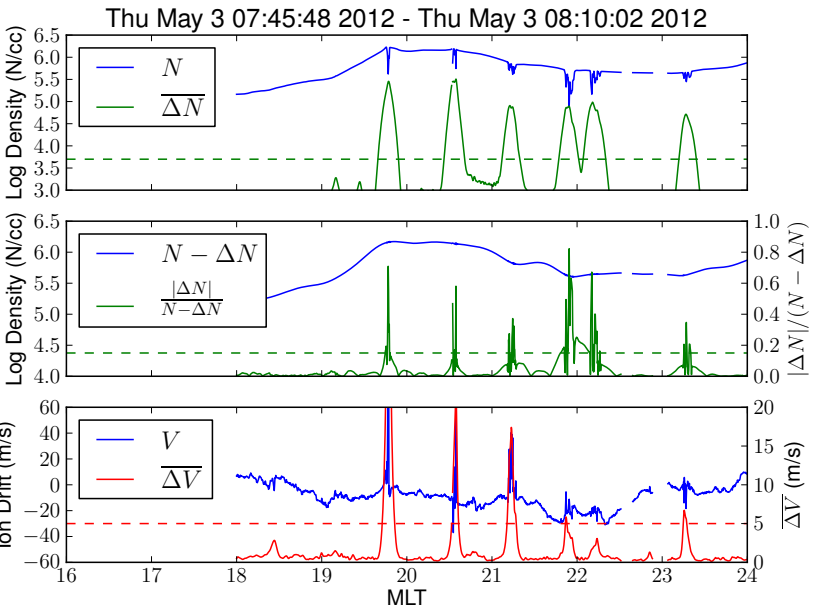

Fig. 2. CINDI measurements of top: ion density and associated variance middle: estimated background ionosphere and the absolute magnitude of the wavelet-derived density perturbations waveform bottom: measurements of meridional ion drift along with its scalelimited variance. A portion of a single orbit is shown.

Table 1. The mean F10.7 cm flux. The December solstice season in 2008/2009 spans from late November 2008 through February 2009. The June solstice in the same column is for 2009, with similar ranges for the remaining years.

\begin{tabular}{lrrrr}
\hline & \multicolumn{4}{c}{ Mean F10.7 Level (sfu) } \\
\hline Season & $2008 / 2009$ & $2009 / 2010$ & $2010 / 2011$ & $2011 / 2012$ \\
Dec Sol. & 69.4 & 78.4 & 85.8 & 135.56 \\
Jun Sol. & 68.8 & 75.5 & 98.5 & 123.8 \\
\hline
\end{tabular}

no trace of the expansion in longitude late at night is observed. Note that $\Delta N / N$ may not identify the presence of an irregularity in the early stages of formation as the density change is not yet significantly different than the background.

The third column is the occurrence of irregularities identified through changes in the meridional ion drift. These distributions are consistent with those obtained by using $\Delta N / N$ for all years. This signature decreases smoothly as solar activity increases each year and is no longer visible by December 2011. The F10.7 values for each season and year are listed in Table 1.

Figure 4 shows the occurrence of irregularities for $\Delta N / N$ and $\overline{\Delta V}$ for the June solstices. The relation between $\overline{\Delta N}$ and $\overline{\Delta V}$ is the same as observed for the December solstices and is not shown. While irregularities are confined to particular longitude sectors at high solar activity, at lower activity levels $\overline{\Delta V}$ and $\Delta N / N$ are observed at almost all longitudes. There is a time lag between the $\overline{\Delta V}$ and $\Delta N / N$ distributions, with $\overline{\Delta V}$ statistically indicating irregularities before $\Delta N / N$. This is expected as in situ measurements and numerical modeling have shown that perturbations in velocity associated with 


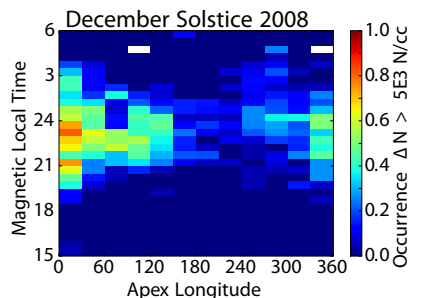

(a)

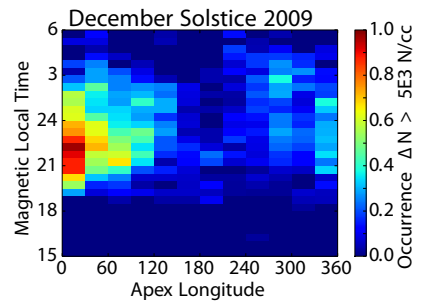

(d)

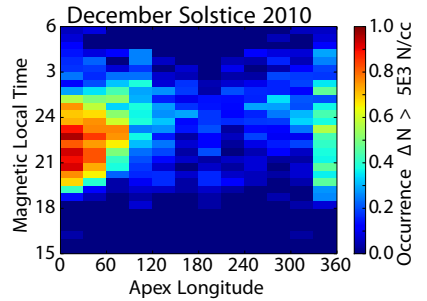

(g)

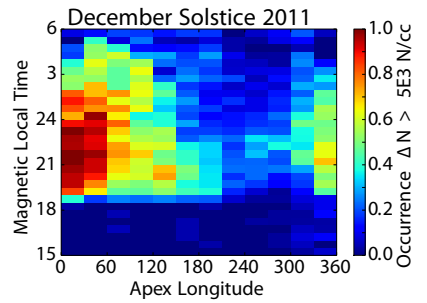

(j)

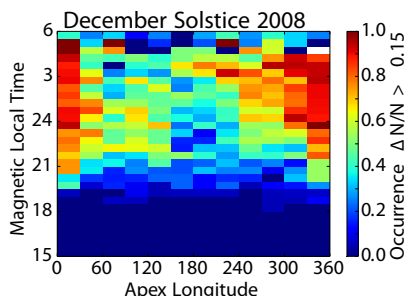

(b)

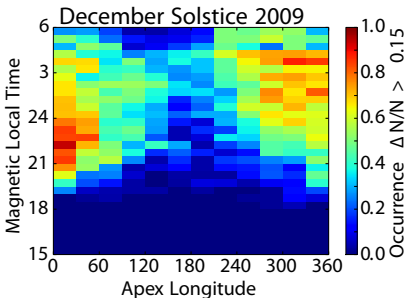

(e)

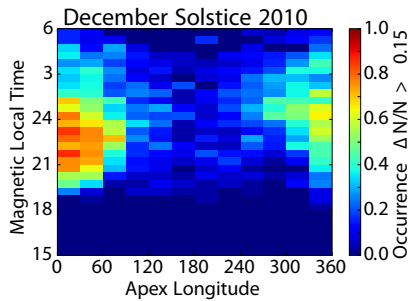

(h)

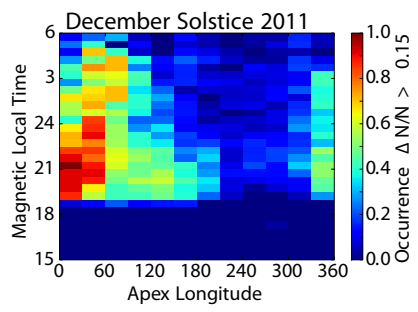

(k)

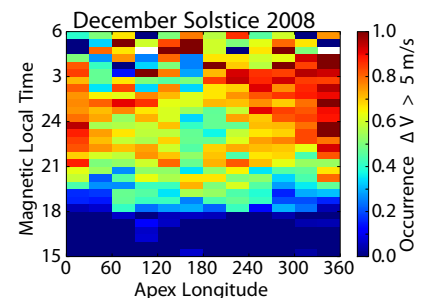

(c)

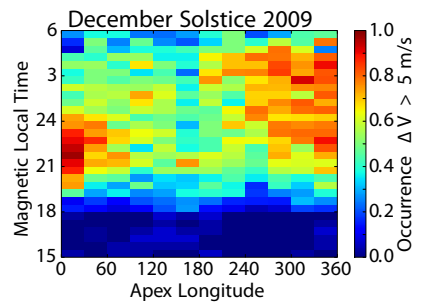

(f)

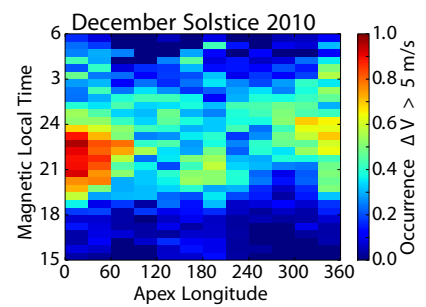

(i)

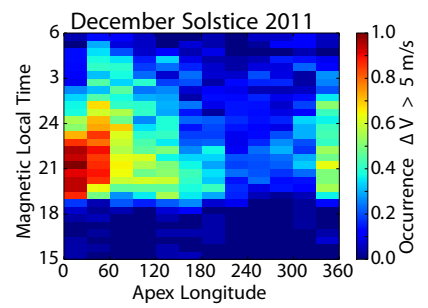

(I)

Fig. 3. Occurrence frequency of detecting ionospheric irregularities with CINDI over the December solstice seasons. Each column varies the wavelet product used, $\Delta N(\mathbf{a}-\mathbf{j}), \Delta N / N(\mathbf{b}-\mathbf{k})$, and $\Delta V(\mathbf{c}-\mathbf{l})$. Each row increments the year, beginning with 2008 for $(\mathbf{a}-\mathbf{c})$ and ending with $2011(\mathbf{j}-\mathbf{l})$. Measurements are restricted to altitudes below $550 \mathrm{~km}$ with $\mathrm{Kp}$ less than $3+$ for the previous $24 \mathrm{~h}$.

an irregularity may be observed before there are changes in plasma density (Aveiro et al., 2012).

The occurrence of scintillation with $S_{4}>0.15$ as estimated by COSMIC measurements in the range of \pm 15 MLAT (magnetic latitude) is shown in Fig. 5. Representative December and June solstices are shown. Other years show similar distributions though there are gaps in the occurrences. A minimum of 10 measurements are required, where the occurrence is the number of observations with significant $S_{4}$ divided by the number of observations. Similar occurrences are seen for CINDI $\Delta N$ and COSMIC in the December solstice, though the CINDI occurrences are a bit lower than the COSMIC threshold. However, the similar longitudinal and local time distribution indicates the in situ irregularities identified by the $\Delta N$ threshold are significant. In later years, all indices give similar distributions in longitude in local time, supporting the thresholds used for $\Delta N / N$ and $\Delta V$. The $\Delta N$ distributions for the June solstice have not been shown; however, the COSMIC pattern compares well with the $\Delta N / N$ and $\Delta V$ patterns in 2011-2012.

The distributions of the equatorial meridional ion drift along with the height of the ionospheric density maximum ( $h m \mathrm{~F} 2$ ) are shown in Figs. 6 and 7 for the December and June solstices, respectively. Measurements for both platforms are restricted to \pm 15 magnetic latitude (MLAT). Variations in the meridional ion drift in the afternoon through sunset are 


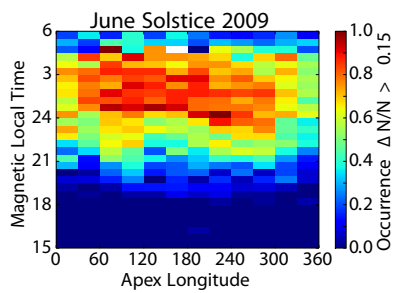

(a)

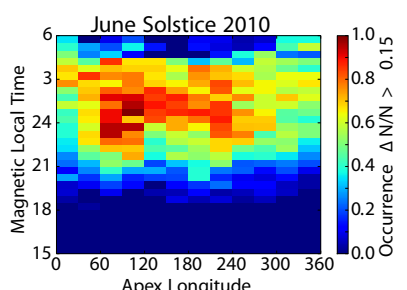

(c)

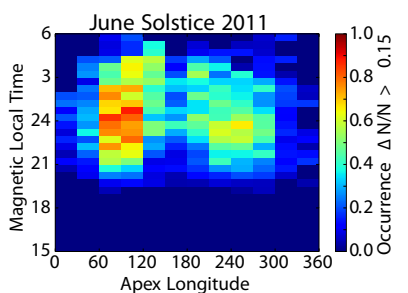

(e)

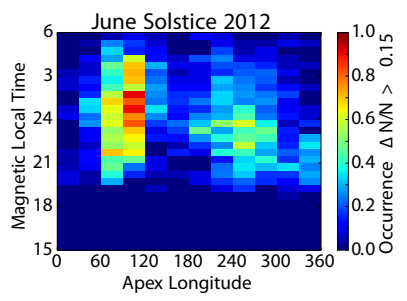

(g)

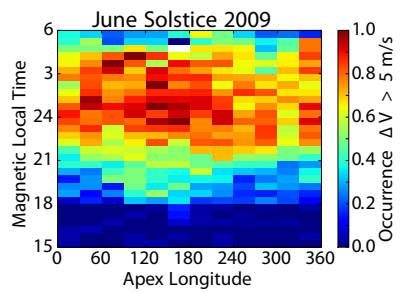

(b)

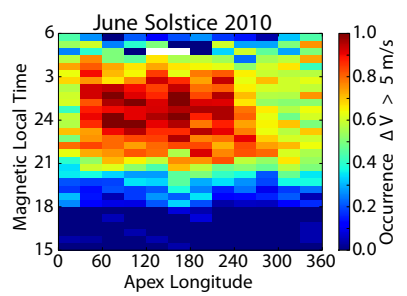

(d)

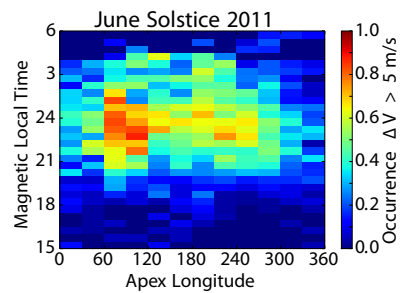

(f)

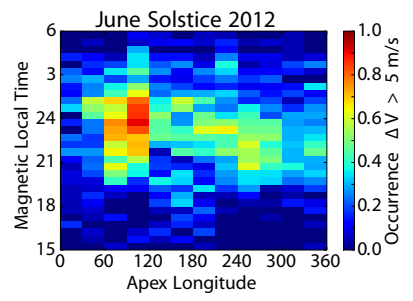

(h)
Fig. 4. Occurrence frequency of detecting irregularities with CINDI over the June solstice seasons. The format follows Fig. 3 though only $\Delta N / N(\mathbf{a}-\mathbf{g})$ and $\Delta V(\mathbf{b}-\mathbf{h})$ are shown.

matched well by the COSMIC $h m \mathrm{~F} 2$. Downturnings in ion drift near $45^{\circ}, 140^{\circ}$ and $240-345^{\circ}$ coincide with decreases in $h m \mathrm{~F} 2$. COSMIC shows similar longitudinal distributions over all years with an increase in $h m \mathrm{~F} 2$ at sunset from 250 $300 \mathrm{~km}$ in 2008 to $380-460 \mathrm{~km}$ in 2011 . The independent agreement between these instruments supports the presented measurements.

Drifts are downward near sunset for December 2008, with a gradual turn towards positive velocities with increasing solar activity. Drifts are generally weak at night, with averages varying between positive and negative in a given sector through the night. Given the day-to-day variability of the ionosphere, drifts in these areas may be up or down on a given date. From 2009 on stronger downward drifts are observed flanking Africa $\left(0-60^{\circ}\right.$ and $\left.120-180^{\circ}\right)$ that increase in magnitude with solar activity. The remaining sectors have

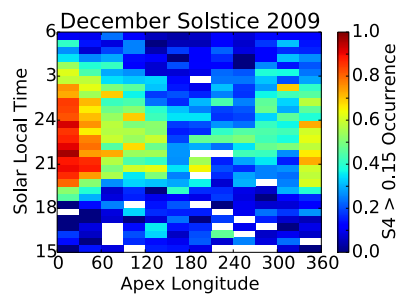

(a)

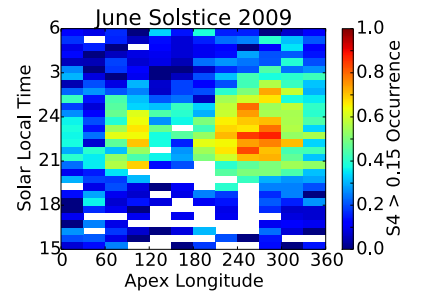

(b)
Fig. 5. COSMIC scintillation occurrence determined from RO data.

weak up or down averages. In 2011 a significant PRE is observed with values in excess of $30 \mathrm{~m} \mathrm{~s}^{-1}$. The return of the PRE is coincident with the appearance of irregularities right after sunset. From 2009 to 2011, there is little change in the longitudinal variation of drifts with longitude other than the return of the PRE.

Correlations between CINDI and COSMIC are also seen near sunset for the June solstice in Fig. 7. Downward drifts on either side of Africa have a corresponding decrease in $h m \mathrm{~F} 2$. The decrease between 180 and $240^{\circ}$ is more visible in the data. Drifts between 0 and $18^{\circ}$ have larger magnitudes in general than the remaining sectors, but the lowest $h m \mathrm{~F} 2$ instances are generally observed between 15 and $105^{\circ}$. Over Africa there is a gradient in $h m \mathrm{~F} 2$ and on average drifts are weak or upward near sunset.

\section{Discussion and conclusion}

The Morlet wavelet has been used to identify ionospheric irregularities through changes in density, normalized density, and meridional ion drift. At the lowest solar activity levels, the normalized density and velocity distributions are highly similar, with active irregularities late into the night and over a broad range of longitudes. This is very different from the $\Delta N$ distribution, with irregularities confined in both longitude and local time. The difference in these indices is a result of the dependence of $\Delta N$ upon the background ionosphere. Irregularities in areas with low density will register as a smaller density perturbation than in areas with larger densities.

The late night low solar activity distributions are seen for both the June and December solstices as well as the equinox seasons (not shown). As solar activity increases, the irregularity distributions for all indices converge on that given by absolute density. The similarity of occurrence magnitudes over all indices for both COSMIC and CINDI at higher solar activity demonstrates the threshold values for each respective quantity are significant and roughly equivalent, though the interpretation of both $\Delta V$ and $\Delta N / N$ does not depend upon the background ionosphere. The use of $\Delta V$ also provides similar information to $\Delta N / N$ without requiring a calculation of the background density. The change in the irregularity 


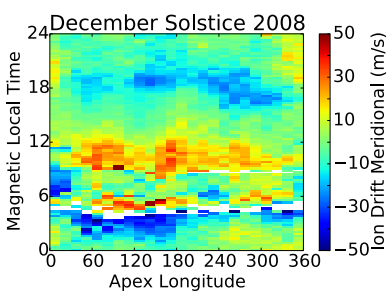

(a)

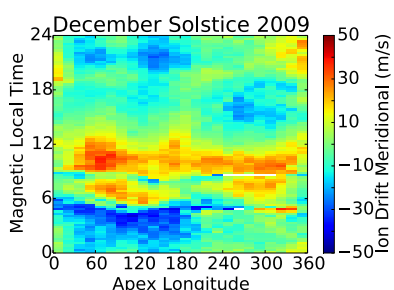

(c)

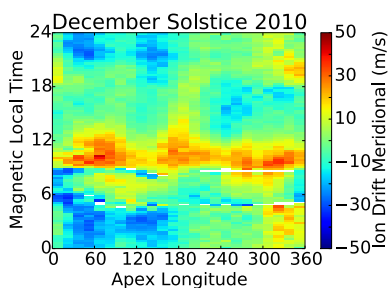

(e)

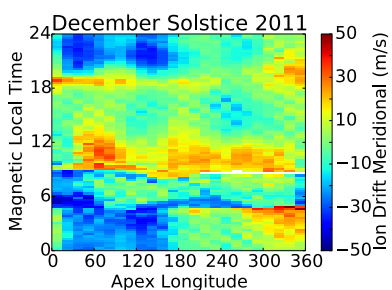

(g)

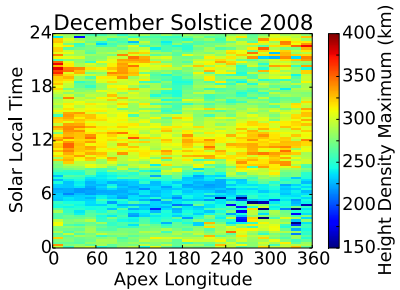

(b)

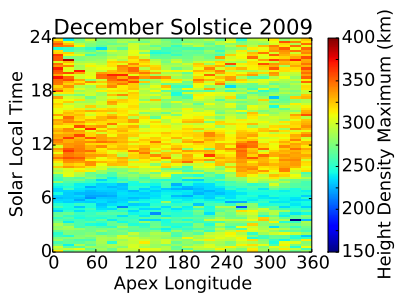

(d)

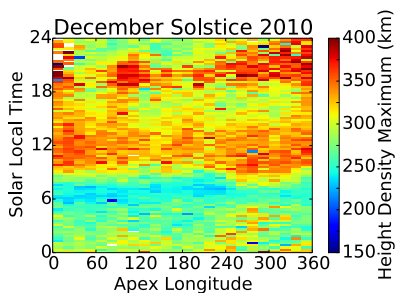

(f)

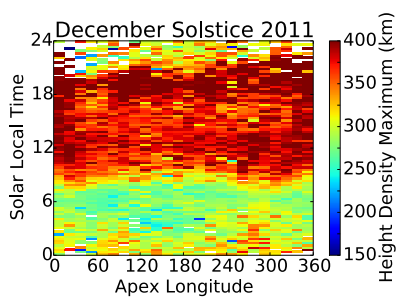

(h)

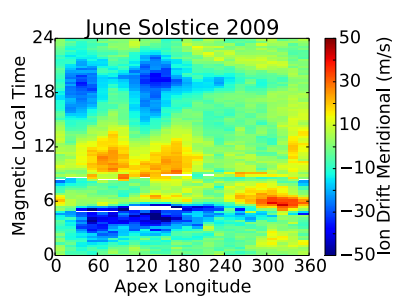

(a)

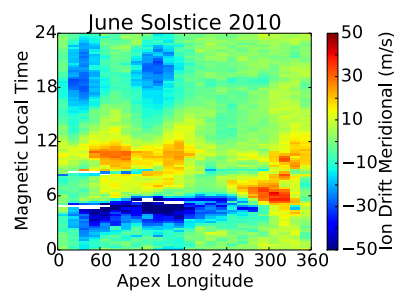

(c)

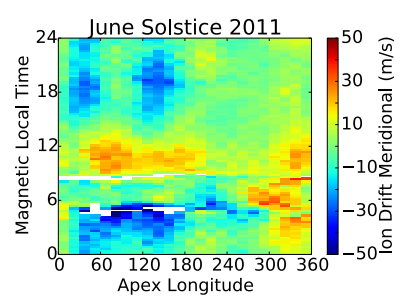

(e)

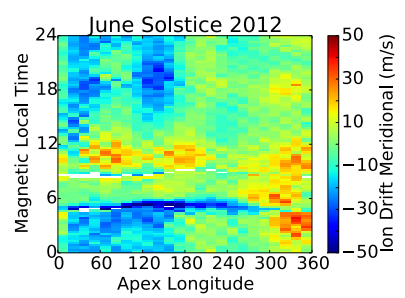

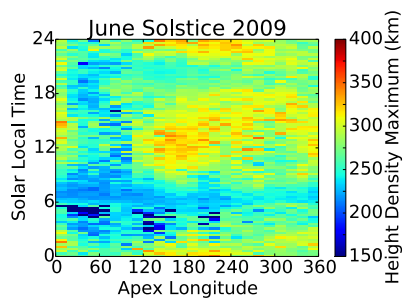

(b)

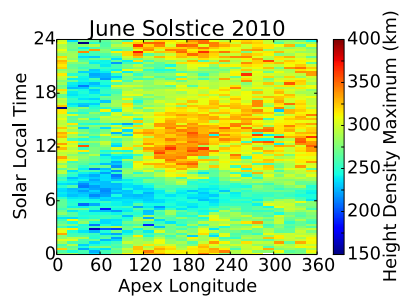

(d)

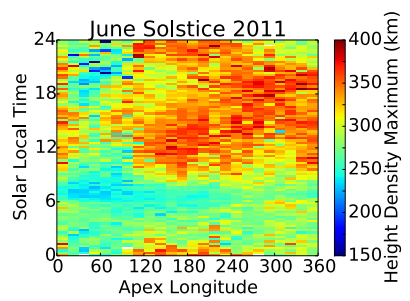

(f)

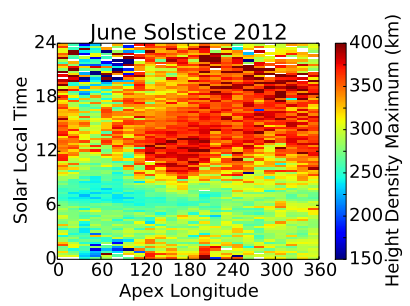

(h)

Fig. 6. Left column: median CINDI meridional ion drifts. Right column: median $h m \mathrm{~F} 2$ near the geomagnetic equator determined by COSMIC.

distribution for these quantities over time confirms a dependence of irregularity formation upon solar activity.

The broad occurrence of irregularities at low solar activity in longitude is not consistent with the hypothesis that the alignment of the solar terminator and the magnetic field at sunset is a controlling factor in irregularity formation. For $\Delta N / N$ at low solar activity, longitudes that are not aligned have irregularities that first appear later in local time near 21:00 MLT and maximize after midnight. However, during moderate solar activities for the December solstice in 2011 irregularities are primarily confined to regions that satisfy this alignment. These irregularities first appear near 19:00 MLT and taper off after midnight for all parameters.

The same general conclusions apply to the June solstice seasons. The broad occurrence of irregularities over all longitudes at low solar activity is not consistent with the alignment hypothesis. As solar activity increases, the longitudinal range

Fig. 7. Left column: median CINDI meridional ion drifts. Right column: median $h m \mathrm{~F} 2$ near the geomagnetic equator determined by COSMIC

contracts, confining irregularity formation to areas with positive declination that are aligned with the terminator. The contraction is observed with both $\Delta V$ and $\Delta N / N$, indices that are not dependent upon the magnitude of the density of the ionosphere.

The expanded distribution of irregularities at low solar activity suggests a change in the relative influence of drivers that lead to the formation of irregularities. Given the relationship between meridional (vertical) ion drifts and the formation of irregularities, changes to this drift could be expected to influence the irregularity occurrence. As expected, a positive relationship is observed between the reappearance of irregularities just after sunset and the PRE in 2011. However, the overall change in the broad longitude response of irregularity formation with solar activity is not matched with similar changes in ion drift. 
The broad longitudinal irregularity occurrence could reflect seeding effects from gravity waves (Abdu, 2012). Work by Vadas and Fritts (2006) found that at solar minimum gravity waves exert forces up to heights of $230 \mathrm{~km}$ and up to $360 \mathrm{~km}$ at solar maximum. While gravity waves do not travel as high in altitude at solar minimum, the force imparted by the gravity waves is larger than at solar maximum. COSMIC measurements demonstrate an increase in $h m \mathrm{~F} 2$ with solar activity, from a range of $250-300 \mathrm{~km}$ in 2008 to $380-460 \mathrm{~km}$ in 2011 during the December solstice, and 230$300 \mathrm{~km}$ in 2009 and $260-380 \mathrm{~km}$ in 2012 during the June solstice. Thus, gravity waves could be expected to exert forces in the bottom-side ionosphere for most seasons presented. Solar activity increases year over year for the presented seasons, reducing the force imparted by a gravity wave source each year. Thus, the expected forcing from gravity waves is consistent with the observed longitudinal and yearly changes in irregularity occurrence.

Fourier analyses of density measurements of irregularities have shown a power law variation with scale size (Livingston et al., 1981; Kil and Heelis, 1998b; Wernik et al., 2007). This power law distribution is a result of the physical processes behind the formation of the irregularity and thus numerous studies have focused on this power spectrum. The successful use of wavelets here to isolate perturbations in both density and velocity enables a comparison of power spectra of simultaneous measurements of density and velocity. An analysis of the relationship between these two spectra as functions of longitude, season, local time, and solar activity may reveal additional information on the formation of irregularities.

Acknowledgements. This work was supported by NASA grant NNX10AT02G.

Topical Editor L. C. Gentile thanks Y.-J. Su and K. Dymond for their help in evaluating this paper.

\section{References}

Abdu, M. A.: Equatorial spread F development and quiet time variability under solar minimum conditions, Indian J. Radio Space, 41, 168-183, 2012.

Abdu, M. A., Bittencourt, J. A., and Batista, I. S.: Magnetic declination control of the equatorial $\mathrm{F}$ region dynamo electric field development and spread F, J. Geophys. Res., 86, 11443-11446, doi:10.1029/JA086iA13p11443, 1981.

Anthes, R. A., Bernhardt, P. A., Chen, Y., Cucurull, L., Dymond, K. F., Ector, D., Healy, S. B., Ho, S. P., Hunt, D. C., Kuo, Y.-H., Liu, H., Manning, K., McCormick, C., Meehan, T. K., Randel, W. J., Rocken, C., Schreiner, W. S., Sokolovskiy, S. V., Syndergaard, S., Thompson, D. C., Trenberth, K. E., Wee, T. K., Yen, N. L., and Zeng, Z.: The COSMIC/FORMOSAT-3 Mission: Early Results, B. Am. Meteorol. Soc., 89, 313-333, doi:10.1175/BAMS-89-3-313, 2008.

Aveiro, H. C., Hysell, D. L., Caton, R. G., Groves, K. M., Klenzing, J., Pfaff, R. F., Stoneback, R., and Heelis, R. A.: Three- dimensional numerical simulations of equatorial spread F: Results and observations in the Pacific sector, J. Geophys. Research., 117, A03325, doi:10.1029/2011JA017077, 2012.

Dymond, K. F.: Global observations of $\mathrm{L}$ band scintillation at solar minimum made by COSMIC, Radio Sci., 47, RS0L18, doi:10.1029/2011RS004931, 2012.

Eccles, J.: Modeling investigation of the evening prereversal enhancement of the zonal electric field in the equatorial ionosphere, J. Geophys. Res., 103, 26709-26719, 1998.

Gentile, L. C., Burke, W. J., and Rich, F. J.: A global climatology for equatorial plasma bubbles in the topside ionosphere, Ann. Geophys., 24, 163-172, doi:10.5194/angeo-24-163-2006, 2006.

Heelis, R. A., Stoneback, R., Earle, G. D., Haaser, R. A., and Abdu, M. A.: Medium-scale equatorial plasma irregularities observed by Coupled Ion-Neutral Dynamics Investigation sensors aboard the Communication Navigation Outage Forecast System in a prolonged solar minimum, J. Geophys. Res., 115, A10321, doi:10.1029/2010JA015596, 2010.

Hei, M. A., Heelis, R. A., and McClure, J. P.: Seasonal and longitudinal variation of large-scale topside equatorial plasma depletions, J. Geophys. Res., 110, A12315, doi:10.1029/2005JA011153, 2005.

Hwang, C., Tseng, T. P., Lin, T. J., Švehla, D., and Hugentobler, U.: Quality assessment of FORMOSAT-3/COSMIC and GRACE GPS observables: analysis of multipath, ionospheric delay and phase residual in orbit determination, GPS solutions, 14, 121131, doi:10.1007/s10291-009-0145-0, 2010.

Kil, H. and Heelis, R. A.: Global distribution of density irregularities in the equatorial ionosphere, J. Geophys. Res., 103, 407-418, doi:10.1029/97JA02698, 1998a.

Kil, H. and Heelis, R. A.: Equatorial density irregularity structures at intermediate scales and their temporal evolution, J. Geophys. Res., 103, 3969-3982, doi:10.1029/97JA03344, 1998 b.

Kil, H., Paxton, L. J., and Oh, S. J.: Global bubble distribution seen from ROCSAT-1 and its association with the evening prereversal enhancement, J. Geophys. Res., 114, 6307, doi:10.1029/2008JA013672, 2009.

Ko, C.: COSMIC/FORMOSAT-3 observations of equatorial F region irregularities in the SAA longitude sector, J. Geophys. Res., 115, A11309, doi:10.1029/2010JA015618, 2010.

Lei, J., Syndergaard, S., Burns, A. G., Solomon, S. C., Wang, W., Zeng, Z., Roble, R. G., Wu, Q., Kuo, Y.-H., Holt, J. M., Zhang, S.-R., Hysell, D. L., Rodrigues, F. S., and Lin, C. H.: Comparison of COSMIC ionospheric measurements with ground-based observations and model predictions: Preliminary results, J. Geophys. Res., 112, A07308, doi:10.1029/2006JA012240, 2007.

Livingston, R., Rino, C., and McClure, J.: Spectral characteristics of medium-scale equatorial F region irregularities, J. Geophys. Res., 86, 2421-2428, 1981.

Schreiner, W. S., Sokolovskiy, S. V., Rocken, C., and Hunt, D. C.: Analysis and validation of GPS/MET radio occultation data in the ionosphere, Radio Sci., 34, 949-966, doi:10.1029/1999RS900034, 2012.

Stolle, C., Lühr, H., and Fejer, B. G.: Relation between the occurrence rate of ESF and the equatorial vertical plasma drift velocity at sunset derived from global observations, Ann. Geophys., 26, 3979-3988, doi:10.5194/angeo-26-3979-2008, 2008.

Stoneback, R., Heelis, R. A., Caton, R. G., Su, Y. J., and Groves, K. M.: In situ irregularity identification and scintillation estima- 
tion using wavelets and CINDI on C/NOFS, Radio Sci., 48, 388395, doi:10.1002/rds.20050, 2013.

Straus, P. R., Anderson, P. C., and Danaher, J. E.: GPS occultation sensor observations of ionospheric scintillation, Geophys. Res. Lett., 30, 1436, doi:10.1029/2002GL016503, 2003.

Su, S. Y., Liu, C. H., Ho, H. H., and Chao, C. K.: Distribution characteristics of topside ionospheric density irregularities: Equatorial versus midlatitude regions, J. Geophys. Res., 111, 6305, doi:10.1029/2005JA011330, 2006.

Su, S. Y., Chao, C. K., and Liu, C. H.: On monthly/seasonal/longitudinal variations of equatorial irregularity occurrences and their relationship with the postsunset vertical drift velocities, J. Geophys. Res., 113, 5307, doi:10.1029/2007JA012809, 2008.

Sultan, P. J.: Linear theory and modeling of the Rayleigh-Taylor instability leading to the occurrence of equatorial spread F, J. Geophys. Res., 101, 26875-26892, doi:10.1029/96JA00682, 1996.

Torrence, C. and Compo, G. P.: A Practical Guide to Wavelet Analysis, B. Am. Meteorol. Soc., 79, 61-78, 1998.

Tsunoda, R. T.: Control of the seasonal and longitudinal occurrence of equatorial scintillations by the longitudinal gradient in integrated Eregion Pedersen conductivity, J. Geophys. Res., 90, 447456, doi:10.1029/JA090iA01p00447, 1985.
Tsyganenko, N. A.: Geopack: A Set of Fortran Subroutines for Computations of the Geomagnetic Field in the Earth's Magnetosphere, available at: http://geo.phys.spbu.ru/ tsyganenko/ Geopack-2008.html (last access: 1 April 2014), 2008.

Vadas, S. L. and Fritts, D. C.: Influence of solar variability on gravity wave structure and dissipation in the thermosphere from tropospheric convection, J. Geophys. Res., 111, A10S12, doi:10.1029/2005JA011510, 2006.

Wernik, A., Alfonsi, L., and Materassi, M.: Scintillation modeling using in situ data, Radio Sci., 42, RS1002, doi:10.1029/2006RS003512, 2007.

Yue, X., Schreiner, W. S., Lei, J., Sokolovskiy, S. V., Rocken, C., Hunt, D. C., and Kuo, Y.-H.: Error analysis of Abel retrieved electron density profiles from radio occultation measurements, Ann. Geophys., 28, 217-222, doi:10.5194/angeo-28-217-2010, 2010. 